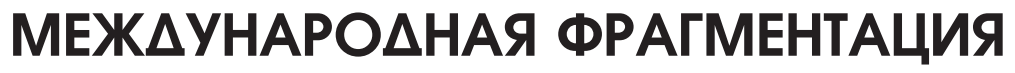

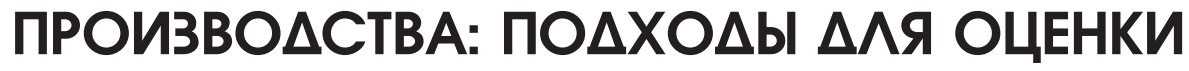

\section{М.Г. Мазитова}

Мазитова Марина Гамиловна - младший научный сотрудник. Институт экономических исследований ДВО РАН, ул. Тихоокеанская, 153, Хабаровск, Россия, 680042. E-mail: mazitova@ecrin.ru.

ORCID: 0000-0002-7978-7904

Аннотаџия. В статье проведена классификация методов исследования международной фрагментации производства в зависимости от источников данных: статистики по давальческой торговле, международной торговле частями и компонентами, таблиц «затраты - выпуск». Проведен сравнительный анализ источников данных и методов исследования. Описаны примеры использования различных методов в зависимости от аспектов исследования. На основе статистики по давальческой торговле можно исследовать только определенные случаи международной фрагментации производства, когда компоненты или материалы вывозят (ввозят) для переработки за рубежом для того чтобы затем реимпортировать (реэкспортировать). Доступность данных международной торговли частями и компонентами и их сопоставимость между странами позволяют проводить анализ мотивов углубления международной фрагментации производства, но недостатком методов является сильная агрегация категорий товарной классификации. Показано, что таблицы «затраты - выпуск», несмотря на ряд методических проблем, являются наиболее предпочтительным источником данных для исследования процессов фрагментации производства, дающим возможность отраслевого анализа, даже если таблицы доступны только для некоторых стран на сопоставимой основе и не обновляются регулярно.

Ключевые слова: международная фрагментация производства, индекс выявленного сравнительного преимущества, RCA, вертикальная специализация, индекс вертикальной специализации, добавленная стоимость

Для ичттирования: Мазитова М.Г. Международная фрагментация производства: подходы для оценки // Пространственная экономика. 2018. № 2. C. 154-169. DOI: 10.14530/ se.2018.2.154-169.

For citation: Mazitova M.G. International Production Fragmentation: Approaches to Measuring. Prostranstvennaya Ekonomika = Spatial Economics, 2018, no. 2, pp. 154-169. DOI: 10.14530/ se.2018.2.154-169. (In Russian).

(C) Мазитова М.Г., 2018 


\section{ВВЕДЕНИЕ}

Международное разделение производственного процесса ${ }^{1}$ и перенесение его частей за границу с использованием местной рабочей силы стали важной особенностью международного разделения труда с середины 1960-х гг. Данный феномен не имеет общепринятого названия. В научной литературе встречаются такие понятия, как фрагментация производства (production fragmentation), вертикальная специализация (vertical specialization), нарезание ломтиков стоимостной цепочки (slicing up the value added chain), аутсорсинг (outsourcing), дезинтеграция производства (disintegration of production), внутрипроизводственная специализация (super-specialization или intra-product specialization) и др.

С усложнением процессов международного разделения труда возникла необходимость в разделении потоков международной торговли на разные составляющие и появлении новых методов учета торговых потоков, связанных с формированием и функционированием международных производственных сетей (international production networks). Благодаря усиливающейся фрагментации при сравнительно умеренном росте производства конечных продуктов стали быстро увеличиваться объемы производства промежуточных товаров и международной торговли ими (Мазитова, Дёмина, 2017). В связи с этим начиная с середины 1980-х гг. в научной литературе появилось большое количество теоретических и эмпирических работ, посвященных исследованию процесса международной фрагментации производства. Основные концептуальные подходы были описаны в 1980-е гг. (Dixit, Grossman, 1982; Sanyal, Jones, 1982 и др.) и 1990-е гг. (Jones, Kierzkowski, 1990; Deardorff, 1998; Yeats, 1998; Venables, 1999 и др.), но с течением времени интерес к данному процессу сохраняется, что обусловлено развитием международных производственных сетей. Совершенствуются существующие и разрабатываются новые методы исследования, например, в работах 2000-х гг. (Hummels, Ishii, Yi, 2001; Kohler, 2004; Grossman, Rossi-Hansberg, 2008 и др.), 2010-х гг. (Johnson, Noguera, 2012; Koopman, Wang, Wei, 2012 и др.).

В эмпирической литературе предлагается большое разнообразие методов исследования международной фрагментации производства в зависимости от того, на каких аспектах фокусируется исследование. В данной статье автором проведена классификация методов исследования в зависимости от используемых источников статистических данных.

${ }^{1}$ В данной статье термины «разделение производственного процесса», «фрагментация производства», «вертикальная специализация» используются как синонимы. 
№ 22018

\section{МЕТОДЫ ИССЛЕДОВАНИЯ МЕЖДУНАРОДНОЙ ФРАГМЕНТАЦИИ ПРОИЗВОДСТВА}

В настоящее время используются следующие основные источники данных для оценки процесса на отраслевом уровне: таможенная статистика по давальческой торговле, статистика международной торговли частями и компонентами, таблицы межотраслевого баланса (Amador, Cabral, 2008b).

Таможенная статистика по давальческой торговле (Custom Statistics on Processing Trade)

Таможенная статистика по давальческой торговле предоставляет информацию о таможенных механизмах, в которых отмена или сокращение тарифов предоставляются в соответствии с предназначением импортируемых товаров. Преимуществом подхода является то, что в таких источниках данных импортируемые товары, которые используются как промежуточные в производственном процессе, идентифицируются однозначно. Такие базы данных используются в ряде эмпирических исследований (Yeats, 1984; Swenson, 2004), но недостатком такого метода является то, что он подходит для описания только определенных случаев международной фрагментации производства, когда компоненты или материалы вывозят (ввозят) для переработки за рубежом для того, чтобы затем реимпортировать (реэкспортировать).

Статистика международной торговли частями и компонентами (International Trade Statistics on Parts and Components)

Товарная классификация международной торговли используется для измерения фрагментации путем сравнения объемов торговли частями и компонентами и объемов торговли готовой продукцией. Доля торговли частями и компонентами является репрезентативной мерой фрагментации. Основное преимущество такого подхода - доступность данных и их сопоставимость между странами, позволяющая идентифицировать конкретные отношения торговых партнеров. Недостатком является то, что этот подход в значительной степени опирается на товарную классификацию торговой статистики. Как правило, совокупность частей и компонентов берется из стандартной классификации международной торговли (SICT) на наиболее детальном уровне и включаются продукты, принадлежащие категориям SICT-7 («Машиностроение и транспортное оборудование») и SICT-8 («Разные готовые изделия»). Данный тип анализа был начат работами А.Д. Йетса (Yeats, 1998), Ф. Ын и А.Д. Йетса (Ng, Yeats, 1999).

Для анализа мотивов углубления международного разделения производства Ф. Ын и А.Д. Йетс предложили использовать индекс выявленно- 
го сравнительного преимущества $\mathrm{RCA}^{1}$, показывающий, имеет ли страна сравнительное преимущество в производстве или сборке частей и компонентов.

Традиционно индекс RCA вычисляется с использованием статистики экспорта и в результате показывает, имеет ли страна сравнительные преимущества в производстве какого-то товара. Индекс выявленного сравнительного преимущества страны $i$ в производстве товара $j$ определяется как

$$
R C A_{i j}^{p}=\frac{x_{i j} / X_{i}}{x_{w j} / X_{w}} \cdot 100,
$$

где $x_{i j}$ - объем экспорта продукта $j$ страны $i ; X_{i}=\sum_{j} x_{i j}$ - объем всего экспорта страны $i ; x_{w j}=\sum_{i} x_{i j}$ - объем мирового экспорта продукта $j$; $X_{w}=\sum_{i} \sum_{j} x_{i j}$ - объем мирового экспорта.

Значение индекса имеет следующую интерпретацию. Если $R C A>1$, то страна $i$ имеет сравнительное преимущество в производстве товара $j$.

Если же индекс RCA вычисляется с использованием статистики импорта частей и компонентов, то он показывает, имеет ли страна преимущества в сборке какого-то товара. Индекс выявленного сравнительного преимущества страны $i$ в сборке товара $j$ определяется как

$$
R C A_{i j}^{a}=\frac{m_{i j} / M_{i}}{m_{w j} / M_{w}} \cdot 100,
$$

где $m$ / $M-$ импорт.

Впоследствии данный вид анализа широко использовался для исследования международной фрагментации производства. Одни авторы акцентировали внимание на конкретных регионах или странах и анализировали в своих работах международную фрагментацию производства с акцентом на опыт Восточной Азии и Китая (Lemoine, Ünal-Kesenci, 2004; Gaulier, Lemoine, Ünal-Kesenci, 2004; Kaminski, Ng, 2001). Другие авторы использовали этот метод для измерения значения фрагментации в конкретных отраслях на примере отдельных стран и географических районов. Например, исследование автомобильного сектора и сектора электроники в Восточной Азии и Латинской Америке (Lall, Albaladejo, Zhang, 2004) показало, что во всем мире процессы производства в электронной промышленности фраг-

${ }^{1}$ RCA (Revealed comparative Advantage) - «выявленное сравнительное преимущество» - показатель конкурентоспособности, определяемый на основе сопоставления показателей внешней торговли страны с показателями других стран, регионов или всего мира. 
ментируются быстрее, чем в автомобильной промышленности, в частности, в Восточной Азии, где сети электронной промышленности являются наиболее развитыми. Ф. Кимура и др. (Kimura, Takahashi, Hayakawa, 2007) pacсматривали структуру международной торговли автомобильными частями и компонентами в Восточной Азии и Европе и сделали вывод, что теория фрагментации объясняет механизм функционирования международных производственных сетей в Восточной Азии.

Таблицы межотраслевого баланса / таблицы «затраты - выпуск» (Input-Output Tables)

Большинство существующих оценок международной фрагментации производства базируются на доле импортируемых факторов производства в валовой продукции, в общих затратах на производство или в экспорте. Как правило, такие измерения проводятся на основе таблиц межотраслевого баланса, иногда дополняемых данными статистики проникновения импорта, вычисляемыми по данным о торговле. Точность измерения фрагментации во многом зависит от существующей товарной классификации. Очень подробная товарная классификация гарантирует, что характеристики производственной цепочки (production chain) определены и отслеживаются должным образом, то есть данный продукт в действительности - промежуточный, используемый в производстве другого продукта. Однако такие данные, как правило, отсутствуют, поэтому недостатком данного метода является сложность осуществления точного межстранового анализа и анализа временных рядов. Выявление стран с показательной офшорной активностью и оценка ее основных тенденций, как правило, проводятся по относительно агрегированной товарной классификации. Основным преимуществом данного метода является возможность проводить временной анализ и анализ по отраслям, даже если таблицы межотраслевого баланса доступны только для некоторых стран на сопоставимой основе и не обновляются регулярно, поэтому этот источник данных считается наиболее подходящим в большинстве исследований.

В эмпирических работах описываются два различных типа измерений, основанных на данных таблиц межотраслевого баланса.

Первый тип измерений фокусируется на иностранной составляющей отечественного производства. Вычисляется доля импортируемых ресурсов в производстве или в общих расходах на производство (Feenstra, Hanson, 1996). Такая мера используется для оценки потенциального воздействия фрагментации на занятость и заработную плату малоквалифицированных работников отечественной экономики. Как правило, результатом таких исследований является подтверждение устойчивого роста масштабов международного аутсорсинга материальных факторов. Так, например, Х.М. Кампа 
и Л. Голдберг обнаружили увеличение доли импортируемых ресурсов для производства в США, Великобритании и Канаде, но не в Японии (Сатра, Goldberg, 1997). А. Хиджен показал, что международный аутсорсинг в Великобритании неуклонно возрастает с начала 1980-х гг, хоть и с различиями в разных отраслях производства (Hijzen, 2005). Исследования Х. Эггера и П. Эггера показали значительный рост австрийского аутсорсинга в Центральной и Восточной Европе в 1990-1998 гг., что отражает снижение торговых барьеров и заработной платы, преобладающей в этих странах (Egger, Egger, 2003).

Второй тип измерений фокусируется на содержании импорта в экспорте (прямом и косвенном), или индексе вертикальной спецчиализации (vertical specialization, VS), предложенном Д. Хуммельсом, Д. Исии и К.-М. Ли (Hummels, Ishii, Yi, 2001). Эта мера непосредственно отражает те случаи, когда производство осуществляется как минимум в двух странах и товары пересекают, по крайней мере дважды, международные границы (puc.).

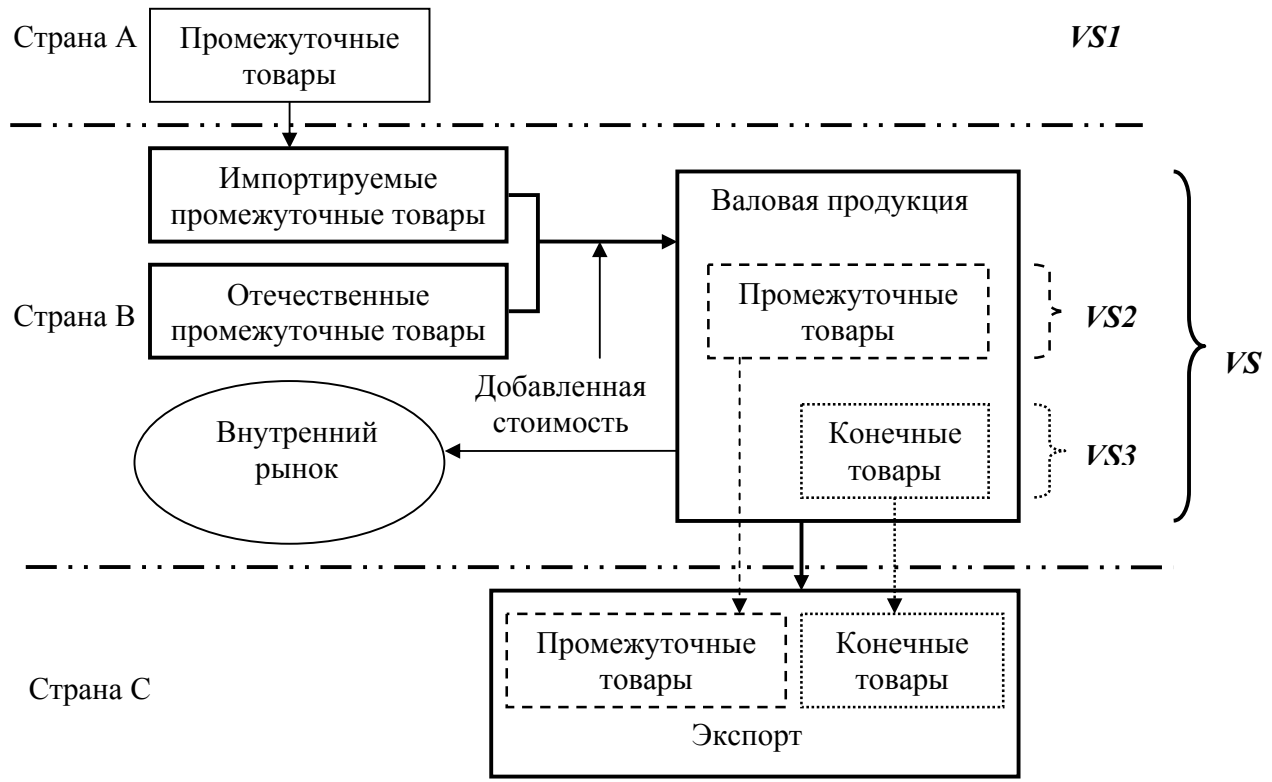

Рuc. Пример вертикальной специализации, включающей три страны

Примечание. Страна А производит промежуточные товары и экспортирует их в страну В. Страна В сочетает импортируемые промежуточные товары с капиталом и рабочей силой (добавленная стоимость) и отечественными промежуточными товарами для производства конечной продукции (валовой продукции), часть которой экспортирует в страну С. Индекс вертикальной специализации VV отражает участие страны В в этом процессе.

Источники: составлено автором на основе: Uchida, 2008; Hummels, Ishii, Yi, 2001.

По сравнению с первой мерой на основе таблиц межотраслевого баланса, которая связана с долей напрямую импортируемых факторов производства 
в валовой продукции, эта мера является более узкой, поскольку добавляется условие, что некоторая итоговая продукция должна быть экспортирована. С другой стороны, измерение вертикальной специализации, предложенное Д. Хуммельсом, Д. Исии и К.-М. Ли, является более широким, так как оно рассматривает также импортируемые факторы производства, косвенно используемые в производстве экспортируемых товаров.

Индекс вертикальной специализации - это содержание импорта в экспорте, или, что эквивалентно, иностранной добавленной стоимости (value added) в экспорте.

$$
\begin{gathered}
V S=\left(\frac{\text { импортируемые промежуточные товарыл }}{\text { валовая продукичя }}\right) \cdot \text { экспорт }= \\
=\left(\frac{\text { экспорт }}{\text { валовая продукция }}\right) \cdot \text { импортируемые промежуточные товары } .
\end{gathered}
$$

Первый множитель в уравнении (1) отражает вклад импортируемых промежуточных товаров в валовую продукцию. Умножая это отношение на величину, которая идет на экспорт, получаем стоимость импортируемой части экспорта. Если страна не использует импортные материалы или если она не осуществляет экспорт своей валовой продукции, то индекс VS равен 0.

Для измерения совокупного индекса вертикальной специализации используют данные по секторам, помечая переменные в уравнениях (1) и (1a) индексами $i$ (сектор) и $k$ (страна). Совокупный индекс VS определяется как сумма соответствующих индексов для каждого сектора:

$$
V S_{k}=\sum_{i} V S_{k i}
$$

Индекс VS страны $k$, выраженный как доля от общего объема экспорта:

$$
\frac{V S_{k}}{X_{k}}=\frac{\sum_{i} V S_{k i}}{\sum_{i} X_{k i}},
$$

где $X$ - экспорт, $k$ - страна, $i$ - сектор, или в матричной форме:

$$
\frac{V S_{k}}{X_{k}}=\frac{u A^{M}\left[I-A^{D}\right]^{-1} X}{X_{k}},
$$

где $u$ - единичный вектор ( $1 \mathrm{x} n) ; A^{M}, A^{D}$ - матрицы $(n \times n)$ коэффициентов затрат на импортные и отечественные товары и услуги соответственно; $I$ - единичная матрица $(n \times \mathrm{x} n) ; X$ - вектор ( $n \times 1)$ экспорта валовой продукции; $X_{k}-$ совокупный экспорт страны $k ; n-$ количество секторов. 
Совокупный индекс VS увеличивается, если использование импортных материалов увеличивается в большом количестве экспортоинтенсивных отраслей и / или если увеличение экспорта в секторах с использованием импортных материалов наиболее интенсивно. Это может произойти, даже если доля промежуточных товаров в импорте падает.

Методика, предложенная Д. Хуммельсом и др., в некоторых случаях с незначительными изменениями оригинальной формулировки, широко применяется в исследованиях вертикальной специализации в различных регионах. Например, Ж. Амадор и С. Кабрал исследовали процессы для Португалии (Amador, Cabral, 2008a), С. Чжан и Д. Сан - для КНP (Zhang, Sun, 2007), X. Чен и Я. Чан - для Тайваня и Республики Корея (Chen, Chang, 2006).

Индекс вертикальной специализации можно разбить на две составляющие: VS2 - индекс, отображающий использование импортируемых промежуточных товаров для производства промежуточных товаров, идущих на экспорт, VS3 - индекс, отображающий использование импортируемых промежуточных товаров для производства конечных товаров, экспортируемых страной. Для вычисления VS2 и VS3 вектор $X$ разбивается на две составляющие - экспорт промежуточных товаров и экспорт конечных товаров.

Индекс VS отражает участие страны В в вертикальной специализации. Можно также оценить участие страны А как долю ее экспорта промежуточных товаров, который был использован в экспорте валовой продукции всех стран-партнеров (VSl) (см. puc.):

$V S 1_{k}=\sum_{j}^{n}$ промежуточные товары, экспортируемые из страны $к$ в страну $j$.

$$
\cdot\left[\frac{\text { экспорт страны } j}{\text { валовая продукция страны } j}\right],
$$

или в матричной форме:

$$
V S 1_{k}=\sum_{j}^{n} u\left[A^{k j}\left[I-A^{j j}\right]^{-1} X_{j}\right] / X_{k},
$$

где $A^{k j}$ - матрица экспорта промежуточных товаров из страны $k$ в страну $j ; A^{j j}-$ матрица коэффициентов отечественных факторов производства страны $j$.

Полная картина вертикальной специализации или позиция страны в вертикально интегрированной производственной сети включает в себя обе меры (VS и $V S 1)$.

Тем не менее индекс VSI труднее измерить, чем $V S$, потому что это требует соответствующих данных о двусторонней торговле в таблицах межотраслевого баланса по всем направлениям, в которые экспортирует продукцию рассматриваемая страна. 
Для измерений, предложенных Д. Хуммельсом и др. (Hummels, Ishii, Yi, 2001), необходимы два основных допущения. Во-первых, интенсивность использования импортируемых ресурсов должна быть одинаковой, вне зависимости от того, производятся ли товары на экспорт или для внутреннего конечного спроса. Это предположение нарушается при увеличении давальческого экспорта, и в экспортируемых товарах используется гораздо большая доля импортных промежуточных факторов производства. Когда используются данные по давальческой торговле, можно не принимать в расчет первое допущение. Р. Купман и др. (Koopman, Wang, Wei, 2008) предложили методику для пересчета внутренней и зарубежной добавленной стоимости в таких случаях.

Второе допущение состоит в том, что все импортируемые промежуточные факторы производства должны содержать 100\% зарубежной добавленной стоимости. То есть, в импорте страны не может быть и косвенной внутренней составляющей. Второе допущение может вообще не выполняться, так как экспорт той или иной страны может содержать материалы, импортированные из множества других стран. Данные международных таблиц межотраслевого баланса позволяют не принимать в расчет второе допущение.

3. Ванг и др. (Wang, Powers, Wei, 2010) предложили рассматривать международную производственную цепочку как распределение долей добавленной стоимости среди стран (регионов) в той или иной отрасли промышленности. В производственной цепочке каждый производитель покупает ресурсы (факторы), а затем добавляет ценность, которая включена в стоимость следующего этапа производства. Сумма добавленной стоимости на каждом этапе в цепочке равна стоимости конечных товаров, производимых в сети. Чтобы точно определить такие цепочки на территории многих стран, необходима количественная оценка вклада каждой страны (региона) в общую добавленную стоимость, произведенную в процессе создания конечной продукции. Международные таблицы межотраслевого баланса позволяют полностью «нарезать» цепочку создания стоимости по всем участвующим в процессе производства странам на среднеотраслевом уровне.

На примере двух стран вся произведенная продукция используется в качестве промежуточных или конечных товаров, потребляемых в стране или за рубежом:

$$
\left[\begin{array}{l}
X^{1} \\
X^{2}
\end{array}\right]=\left[\begin{array}{cc}
I-A_{11} & -A_{12} \\
-A_{21} & I-A_{22}
\end{array}\right]^{-1}\left[\begin{array}{l}
Y^{1} \\
Y^{2}
\end{array}\right]=\left[\begin{array}{ll}
B_{11} & B_{12} \\
B_{21} & B_{22}
\end{array}\right]\left[\begin{array}{l}
Y^{1} \\
Y^{2}
\end{array}\right],
$$

где $X^{r}$ - вектор $(n \times 1)$ валовой продукции страны $r ; Y^{r}$ - вектор $(n \times 1)$ конечного спроса, включающего внутренний конечный спрос и экспорт конечной 
продукции страны $r ; A_{s r}$ - матрица $(n \times n)$ коэффициентов международной таблицы «затраты - выпуск» - использование в стране $r$ промежуточных товаров из страны $s ; B_{s r}-$ блок обратной матрицы Леонтьева $(n \mathrm{x} n)$, которая является матрицей коэффициентов полных затрат, дает количество общего объема производства в стране-производителе $s$, требующееся для увеличения на единицу конечного спроса в стране $r$.

Тогда добавленная стоимость в экспорте каждого сектора (value added by source in gross exports):

$$
V A S_{-} E=\left[\begin{array}{cc}
V_{1} B_{11} E^{12} & V_{1} B_{12} E^{21} \\
V_{2} B_{21} E^{12} & V_{2} B_{22} E^{21}
\end{array}\right],
$$

где $V_{s}$ - вектор (1 х $\left.n\right)$ коэффициентов прямой добавленной стоимости; $E^{s r}$ - вектор ( $n$ x 1) официального валового экспорта, включающего экспорт как промежуточных, так и конечных товаров из страны $s$ в страну $r$.

Диагональные элементы в матрице (2) определяют долю внутренней добавленной стоимости в единице экспорта страны. Недиагональные элементы дают значение зарубежной добавленной стоимости, включенной в единицу экспорта страны (что соответствует индексам VS1 и VS, предложенным Д. Хуммельсом и др.).

Межстрановые межотраслевые балансы (Inter-Country Input-Output Model, ICIO) и статистика торговли добавленной стоимостью (Trade in Value-Added, TiVA)

В последние годы практически все экономические процессы выстраиваются вокруг региональных и глобальных производственных сетей. В связи с этим совершенствуются статистика международной торговли и методы исследования международной фрагментации производства. Место страны в международном разделении труда в настоящее время определяется в большей степени не номенклатурой экспортируемой продукции, а теми функциями, которые страна выполняет в процессе производства товаров и оказания услуг. В статистике международной торговли стали учитывать не только таможенную стоимость товара, но и стоимость, добавленную обработкой внутри страны, что позволяет определить вклад различных стран в изготовление конечного товара.

В научной литературе особое внимание уделяется исследованию глобальных цепочек добавленной стоимости (global value chains) и определению степени вовлеченности стран в них (Пономаренко, Мурадов, 2014; Кондратьев, 2015; Соколов, 2016). Исследования проводятся с использованием базы данных ОЭСР-ВТО «Торговля добавленной стоимостью» (TiVA) и различных индикаторов, разработанных и рассчитанных на основе этой базы (TiVA 2016 indicators - definitions). Статистика торговли добавленной 
стоимости рассчитывается на основе межстрановых межотраслевых балансов (ICIO), связывающих воедино международные межотраслевые балансы 63 стран. В действительности использование в исследованиях баз данных ICIO и TiVA не предполагает каких-то новых методов исследования, отличающихся от методов на основе национальных таблиц «затраты - выпуск», но новые базы данных позволяют сократить ряд методологических проблем (например, связанных с качеством статистических данных по международной торговле и производству) и упростить расчеты в исследованиях.

\section{ЗАКЛЮЧЕНИЕ}

Методы исследования международной фрагментации производственного процесса отличаются в зависимости от данных, используемых для анализа.

Таможенная статистика по давальческой торговле предоставляет информацию о таможенных механизмах, в которых отмена или сокращение тарифов предоставляются в соответствии с предназначением импортируемых товаров. Преимуществом подхода является то, что в таких источниках данных импортируемые товары, которые используются как промежуточные в производственном процессе, идентифицируются однозначно. Недостатком такого метода является то, что он подходит для описания только определенных случаев международной фрагментации производства, когда компоненты или материалы вывозят (ввозят) для переработки за рубежом, для того чтобы затем реимпортировать (реэкспортировать).

Статистика международной торговли частями и компонентами на основе товарной классификации международной торговли используется для измерения степени фрагментации производства путем сравнения объемов торговли частями и компонентами и объемов торговли готовой продукцией. Основным преимуществом такого подхода является доступность данных и их сопоставимость между странами, позволяющая идентифицировать конкретные отношения торговых партнеров. Недостатком является то, что он в значительной степени опирается на товарную классификацию торговой статистики, категории которой зачастую сильно агрегированы.

Большинство существующих оценок международной фрагментации производства базируются на доле импортируемых факторов производства в валовой продукции страны, в общих затратах на производство или в экспорте. Как правило, такие измерения проводятся на основе таблиц межотраслевого баланса, дополняемых иногда данными статистики проникновения импорта. Точность измерения фрагментации во многом зависит от существующей товарной классификации. Для точных оценок необходима 
очень подробная товарная классификация, однако такие данные, как правило, отсутствуют, поэтому недостатком данного метода является сложность осуществления точного межстранового анализа и анализа временных рядов. Выявление стран с показательной офшорной активностью и оценка ее основных тенденций, как правило, проводятся по относительно агрегированной товарной классификации. Основным преимуществом данного метода является возможность проводить временной анализ и анализ по отраслям, даже если таблицы межотраслевого баланса доступны только для некоторых стран на сопоставимой основе и не обновляются регулярно. К тому же в последнее время разрабатываются комплексные межстрановые межотраслевые балансы, включающие данные по все большему количеству странпартнеров и отраслям производства, что позволяет сократить ряд проблем данного метода.

\section{СПИСОК ЛИТЕРАТУРЫ}

Кондратьев В.Б. Мировая экономика как система глобальных цепочек стоимости // Мировая экономика и международные отношения. 2015. № 3. С. 5-17.

Мазитова М.Г., Дёмина Я.В. Международные производственные сети: роль стран Восточной Азии // Пространственная экономика. 2017. № 1. С. 71-98. DOI: 10.14530/se.2017.1.071-098

Пономаренко А.Н., Мурадов К.Ю. Новая статистика движения добавленной стоимости в международной торговле // Экономический журнал ВШЭ. 2014. Т. 18. № 1. C. $43-79$.

Соколов В.В. Международные производственные цепочки: учет вклада стран в создание добавленной стоимости // Российский внешнеэкономический вестник. 2016. № 2. C. 48-59.

Amador J., Cabral S. The Portuguese Export Performance in Perspective: A Constant Market Share Analysis // Economic Bulletin. 2008a.

Amador J., Cabral S. Vertical Specialization across the World: A Relative Measure // Banco de Portugal Working Papers. 2008b. No. 10-2008. Pp. 1-28. URL: https://mpra.ub.unimuenchen.de/9618/1/MPRA_paper_9618.pdf (дата обращения: апрель 2018).

Campa J., Goldberg L.S. The Evolving External Orientation of Manufacturing: A Profile of Four Countries // Federal Reserve Bank of New York Economic Policy Review. 1997. Vol. 3. Issue 2. Pp. 53-81.

Chen H.-Y., Chang Y.-M. Trade Verticality and Structural Change in Industries: The Cases of Taiwan and South Korea // Open Economies Review. 2006. Vol. 17. Issue 3. Pp. 321-340.

Deardorff A.V. Fragmentation across Cones / Michigan-Center for Research on Economic \& Social Theory Papers. 1998.

Dixit A.K., Grossman G.M. Trade and Protection with Multistage Production // The Review of Economic Studies. 1982. Vol. 49. Issue 4. Pp. 583-594. DOI: 10.2307/2297288.

Egger H., Egger P. Outsourcing and Skill-Specific Employment in a Small Economy: Austria after the Fall of the Iron Curtain // Oxford Economic Papers. 2003. Vol. 55. Issue 4. Pp. 625-643. DOI: 10.1093/oep/55.4.625. 
Feenstra R.C., Hanson G.H. Globalization, Outsourcing and Wage Inequality // American Economic Review. 1996. Vol. 86. Issue 2. Pp. 240-245.

Gaulier G., Lemoine F., Ünal-Kesenci D. China's Integration in Asian Production Networks and its Implications / RIETI Discussion Paper 04-E-033. 2004. URL: https://www.rieti. go.jp/jp/publications/dp/04e033.pdf (дата обращения: май 2018).

Grossman G.M., Rossi-Hansberg E. Trading Tasks: A Simple Theory of Offshoring // American Economic Review. 2008. Vol. 98. No. 5. Pp. 1978-1997. DOI: 10.1257/ aer.98.5.1978.

Hijzen A. A Bird's Eye View of International Outsourcing: Data, Measurement and Labour Demand Effects // Économie internationale. 2005. Vol. 104. No. 4. Pp. 45-63.

Hummels D., Ishii J., Yi K.-M. The Nature and Growth of Vertical Specialization in World Trade // Journal of International Economics. 2001. Vol. 54. Issue 1. Pp. 75-96. DOI: 10.1016/S0022-1996(00)00093-3.

Hummels D., Rapoport D., Yi K.-M. Vertical Specialization and the Changing Nature of World Trade // Federal Reserve Bank of New York Economic Policy Review. 1998. Vol. 4. Issue 2. Pp. 79-99.

Johnson R.C., Noguera G. Proximity and Production Fragmentation // The American Economic Review. 2012. Vol. 102. No. 3. Pp. 407-411. DOI: 10.1257/aer.102.3.407.

Jones R.W., Kierzkowski H. The Role of Services in Production and International Trade: A Theoretical Framework / RCER Working Papers. 1988.

Kaminski B., $\mathrm{Ng} \mathrm{F}$. Trade and Production Fragmentation: Central European Economies in European Union Networks of Production and Marketing / Policy Research Working Paper 2611. 2001. World Bank.

Kimura F., Takahashi Y., Hayakawa K. Fragmentation and Parts and Components Trade: Comparison between East Asia and Europe // The North American Journal of Economics and Finance. 2007. Vol. 18. Issue 1. Pp. 23-40. DOI: 10.1016/j.najef.2006.12.002.

Kohler W. Aspects of International Fragmentation // Review of International Economics. 2004. Vol. 12. Issue 5. Pp. 793-816. DOI: 10.1111/j.1467-9396.2004.00482.x.

Koopman R., Wang Z., Wei S. How Much Chinese Exports is Really Made in China? Assessing Foreign and Domestic Value-Added in Gross Exports / Office of Economics Working Paper No. 2008-03-B. 2008. URL: https://www.usitc.gov/publications/332/ ec200803b revised.pdf (дата обращения: апрель 2018).

Koopman R., Wang Z., Wei S.-J. Tracing Value-added and Double Counting in Gross Exports / National Bureau of Economic Research Working Papers 18579. 2012. URL: http://www.nber.org/papers/w18579.pdf (дата обращения: апрель 2018).

Lall S., Albaladejo M., Zhang J. Mapping Fragmentation: Electronics and Automobiles in East Asia and Latin America // Oxford Development Studies. 2004. Vol. 32. Issue 3. Pp. 407-432. DOI: 10.1080/1360081042000260601.

Lemoine F., Unal-Kesenci D. Assembly Trade and Technology Transfer: The Case of China // World Development. 2004. Vol. 32. Issue 5. Pp. 829-850. DOI: 10.1016/j. worlddev.2004.01.001.

$\mathrm{Ng}$ F., Yeats A.J. Production Sharing in East Asia: Who Does What for Whom, and Why? // World Bank Policy Research Working Paper 2197. 1999.

Sanyal K.K., Jones R.W. The Theory of Trade in Middle Products // The American Economic Review. 1982. Pp. 16-31.

Swenson D.L. Overseas Assembly and Country Sourcing Choices / NBER Working Paper No. 10697. August 2004. URL: http://www.nber.org/papers/w10697.pdf (дата обращения: апрель 2018).

Uchida Y. Vertical Specialization in East Asia: Some Evidence from East Asia Using Asian International Input-Output Tables from 1975 to 2000 // Vertical Specialization and 
Economic Integration in East Asia / Edited by D. Hiratsuka, Y. Uchida. IDE-JETRO. 2008. Pp. 1-28. URL: http://www.ide.go.jp/library/English/Publish/Download/ Report/2007/pdf/2007_01_08_01.pdf (дата обращения: апрель 2018).

Venables A.J. Fragmentation and Multinational Production // European Economic Review. 1999. Vol. 43. Issue 4. Pp. 935-945. DOI: 10.1016/S0014-2921(98)00106-8.

Wang Z., Powers W., Wei S. Value Chains in East Asian Production Networks: An International Input-Output Model Based Analysis / USITC Working Paper No. 2009-10-C. 2009.

Yeats A.J. Just How Big is Global Production Sharing? / The World Bank Policy Research Working Paper Series 1871. 1998.

Zhang X., Sun J. An Analysis of China's Global Industrial Competitive Strength Based on Vertical Specialization // Frontiers of Economics in China. 2007. Vol. 2. Issue 1. Pp. 57-73. DOI: 10.1007/s11459-007-0003-3.

TiVA 2016 indicators - definitions / OECD. 2017. URL: http://www.oecd.org/sti/ind/tiva/ TIVASaM_2016_Indicator_Definitions.pdf (дата обращения: май 2018).

\title{
INTERNATIONAL PRODUCTION FRAGMENTATION: APPROACHES TO MEASURING
}

\author{
M.G. Mazitova
}

Marina Gamilovna Mazitova - Junior Research Fellow. Economic Research Institute FEB RAS, 153 Tikhookeanskaya Street, Khabarovsk, Russia, 680042. E-mail: mazitova@ecrin.ru.

ORCID: 0000-0002-7978-7904

Abstract. The article presents the classification of study methods of international production fragmentation depending on the source of data: custom statistics on processing trade, international trade on parts and components, input-output tables. The author conducted comparative analysis of data sources and study methods and described the examples of using various methods depending on the aspects of research. Based on the statistics of processing trade one can study only certain cases of international production fragmentation, when components or materials are exported (imported) to be processed abroad and then re-imported (re-exported). The availability of data on international trade of parts and components and its comparability between countries allows conducting an analysis of expansion motives of international production fragmentation, but the drawback of the methods is strong aggregation of categories of goods classification. Input-output tables, despite an array of methodical issues, are the most preferable source of data for studying the processes of production fragmentation that allows for industrial analysis, even if the tables are available for certain countries only on comparative basis and are not updated regularly. The article shows example of new improved sources of data (complex intercountry input-output tables, trade in value added), which allow softening the issues of methods based on national input-output tables.

Keywords: international production fragmentation, revealed comparative advantage, RCA, vertical specialization, vertical specialization index, value added 
Kondrat'ev V.B. World Economy as Global Value Chain's Network. Mirovaya Ekonomika i Mezhdunarodnye Otnosheniya = World Economy and International Relations, 2015, no. 3, pp. 5-17. (In Russian).

Mazitova M.G., Dyomina Ya.V. International Production Networks: The Role of East Asia. Prostranstvennaya Ekonomika = Spatial Economics, 2017, no. 1, pp. 71-98. DOI: 10.14530/se.2017.1.071-098. (In Russian).

Ponomarenko A.N., Muradov K.Yu. New Statistics of International Trade in Value Added Terms. Ekonomicheskiy Zhurnal VSHE = HSE Economic Journal, 2014, vol. 18, no. 1, pp. 43-79. (In Russian).

Sokolov V.V. Global Value Chains: Accounting for the Participation of IndividualCountries in the Creation of Value Added. Rossiyskiy Vneshneekonomicheskiy Vestnik = Russian Foreign Economic Journal, 2016, no. 2, pp. 48-59. (In Russian).

Amador J., Cabral S. The Portuguese Export Performance in Perspective: A Constant Market Share Analysisю. Economic Bulletin, 2008a.

Amador J., Cabral S. Vertical Specialization across the World: A Relative Measure. Banco de Portugal Working Papers, 2008b, no. 10-2008. Pp. 1-28. Available at: https://mpra. ub.uni-muenchen.de/9618/1/MPRA_paper_9618.pdf (accessed April 2018).

Campa J., Goldberg L.S. The Evolving External Orientation of Manufacturing: A Profile of Four Countries. Federal Reserve Bank of New York Economic Policy Review, 1997, vol. 3, issue 2, pp. 53-81.

Chen H.-Y., Chang Y.-M. Trade Verticality and Structural Change in Industries: The Cases of Taiwan and South Korea. Open Economies Review, 2006, vol. 17, issue 3, pp. 321-340.

Deardorff A.V. Fragmentation across Cones. Michigan-Center for Research on Economic \& Social Theory Papers, 1998.

Dixit A.K., Grossman G.M. Trade and Protection with Multistage Production. The Review of Economic Studies, 1982, vol. 49, issue 4, pp. 583-594. DOI: 10.2307/2297288.

Egger H., Egger P. Outsourcing and Skill-Specific Employment in a Small Economy: Austria after the Fall of the Iron Curtain. Oxford Economic Papers, 2003, vol. 55, issue 4, pp. 625-643. DOI: 10.1093/oep/55.4.625

Feenstra R.C., Hanson G.H. Globalization, Outsourcing and Wage Inequality. American Economic Review, 1996, vol. 86, issue 2, pp. 240-245.

Gaulier G., Lemoine F., Ünal-Kesenci D. China's Integration in Asian Production Networks and its Implications. RIETI Discussion Paper 04-E-033, 2004. Available at: https:// www.rieti.go.jp/jp/publications/dp/04e033.pdf) (accessed May 2018).

Grossman G.M., Rossi-Hansberg E. Trading Tasks: A Simple Theory of Offshoring. American Economic Review, 2008, vol. 98, no. 5, pp. 1978-1997. DOI: 10.1257/aer.98.5.1978.

Hijzen A. A Bird's Eye View of International Outsourcing: Data, Measurement and Labour Demand Effects. Économie Internationale, 2005, vol. 104, no. 4, pp. 45-63.

Hummels D., Ishii J., Yi K.-M. The Nature and Growth of Vertical Specialization in World Trade. Journal of International Economics, 2001, vol. 54, issue 1, pp. 75-96. DOI: 10.1016/S0022-1996(00)00093-3.

Hummels D., Rapoport D., Yi K.-M. Vertical Specialization and the Changing Nature of World Trade. Federal Reserve Bank of New York Economic Policy Review, 1998, vol. 4, issue 2, pp. 79-99.

Johnson R.C., Noguera G. Proximity and Production Fragmentation. The American Economic Review, 2012, vol. 102, no. 3, pp. 407-411. DOI: 10.1257/aer.102.3.407.

Jones R.W., Kierzkowski H. The Role of Services in Production and International Trade: A Theoretical Framework. RCER Working Papers. 1988. 
Kaminski B., Ng F. Trade and Production Fragmentation: Central European Economies in European Union Networks of Production and Marketing. Policy Research Working Paper 2611, 2001, World Bank.

Kimura F., Takahashi Y., Hayakawa K. Fragmentation and Parts and Components Trade: Comparison between East Asia and Europe. The North American Journal of Economics and Finance, 2007, vol. 18, issue 1, pp. 23-40. DOI: 10.1016/j.najef.2006.12.002.

Kohler W. Aspects of International Fragmentation. Review of International Economics, 2004, vol. 12, issue 5, pp. 793-816. DUI: 10.1111/j.1467-9396.2004.00482.x.

Koopman R., Wang Z., Wei S. How Much Chinese Exports is Really Made in China? Assessing Foreign and Domestic Value-Added in Gross Exports. Office of Economics Working Paper No. 2008-03-B, 2008. Available at: https://www.usitc.gov/publications/332/ ec200803b_revised.pdf (accessed April 2018).

Koopman R., Wang Z., Wei S.-J. Tracing Value-added and Double Counting in Gross Exports. National Bureau of Economic Research Working Papers 18579, 2012. Available at: http://www.nber.org/papers/w18579.pdf (accessed April 2018).

Lall S., Albaladejo M., Zhang J. Mapping Fragmentation: Electronics and Automobiles in East Asia and Latin America. Oxford Development Studies, 2004, vol. 32, issue 3, pp. 407-432. DOI: 10.1080/1360081042000260601.

Lemoine F., Ünal-Kesenci D. Assembly Trade and Technology Transfer: The Case of China. World Development, 2004, vol. 32, issue 5, pp. 829-850. DOI: 10.1016/j.worlddev.2004.01.001.

Ng F., Yeats A.J. Production Sharing in East Asia: Who Does What for Whom, and Why? World Bank Policy Research Working Paper 2197, 1999.

Sanyal K.K., Jones R.W. The Theory of Trade in Middle Products. The American Economic Review, 1982, pp. 16-31.

Swenson D.L. Overseas Assembly and Country Sourcing Choices. NBER Working Paper No. 10697, August 2004. Available at: http://www.nber.org/papers/w10697.pdf (accessed April 2018).

Uchida Y. Vertical Specialization in East Asia: Some Evidence from East Asia Using Asian International Input-Output Tables from 1975 to 2000. Vertical Specialization and Economic Integration in East Asia. Edited by D. Hiratsuka, Y. Uchida. IDE-JETRO, 2008, pp. 1-28. Available at: http://www.ide.go.jp/library/English/Publish/Download/Report/2007/pdf/2007_01_08_01.pdf (accessed April 2018).

Venables A.J. Fragmentation and Multinational Production. European Economic Review, 1999, vol. 43, issue 4, pp. 935-945. DOI: 10.1016/S0014-2921(98)00106-8.

Wang Z., Powers W., Wei S. Value Chains in East Asian Production Networks: An International Input-Output Model Based Analysis. USITC Working Paper No. 200910-C, 2009.

Yeats A.J. Just How Big is Global Production Sharing? The World Bank Policy Research Working Paper Series 1871, 1998.

Zhang X., Sun J. An Analysis of China's Global Industrial Competitive Strength Based on Vertical Specialization. Frontiers of Economics in China, 2007, vol. 2, issue 1, pp. 57-73. DOI: 10.1007/s11459-007-0003-3.

TiVA 2016 Indicators - Definitions. OECD, 2017. Available at: http://www.oecd.org/sti/ind/ tiva/TIVASaM_2016_Indicator_Definitions.pdf (accessed May 2018). 